\title{
Notas sobre el modelo urbano latinoamericano
}

\author{
antonio Zarate Martín *
}

La magnitud del proceso de urbanización de Latinoamérica en la actualidad, la dimensión de los problemas que suscita, en un contexto de profunda crisis estructural resultante de las propias contradicciones generadas por el capitalismo periférico, y las expectativas de transformación y cambio social y económico que hoy abre, motivan nuestro interés por el análisis del modelo urbano latinoamericano. Para ello nos hemos valido de la utilización del índice Rn, como medida de distribución espacial de los asentamientos, y del índice de primacía y de la regla rango tamaño, como medidas de observación de la jerarquía urbana, asi como de su representación cartográfica y gráfica.

\section{UNAS ELEVADAS TASAS DE URBANIZACIÓN}

En un planeta de ciudades que avanza hacia una verdadera ecumenópolis, favorecido por el proceso de convergencia espacio-tiempo, derivado de la revolución tecnológica de las comunicaciones, lo primero que destaca en el panorama urbano de América Latina son los elevados indices de urbanización, sobre todo si se comparan con los correspondientes a países en vías de desarrollo de otros conjuntos regionales. Hoy,

Departamento de Geografía, Universidad Nacional de Educación a Distancia, Ciudad Universitaria, 28071 MADRID, España. 
más del $67 \%$ de la población latinoamericana vive en ciudades, mientras que en Asia lo hace el $31 \%$ y en África, el $30 \%$. Para el año 2000 se estima que unos 550 millones de personas, el $77 \%$ de los latinoamericanos, residirán en áreas urbanas ${ }^{1}$ (Figura 1).

La tasa global de urbanización es semejante a la de Europa en los años setenta y Norteamérica en los cincuenta, y aunque existen considerables diferencias dentro de Latinoamérica, del $55 \%$ de población urbana en el Caribe al $84,3 \%$ en el Cono Sur en 1985, todas sus subregiones son predominantemente urbanas.

Estas elevadas tasas de urbanización son resultado de la aceleración del proceso de urbanización de América Latina después de la Segunda Guerra Mundial y de la tradición del fenómeno urbano en este ámbito espacial a diferencia de los de otras regiones del mundo.

Las dimensiones del proceso reciente de urbanización se ponen de relieve si consideramos que en 1950 sólo había 66 ciudades latinoamericanas de más de cien mil habitantes y una de más de cinco millones, mientras que ya en 1980 había 245 de más de cien mil habitantes y cinco superaban los cinco millones. Hoy, existen 25 ciudades de más de un millón de habitantes; juntas reúnen 93 millones de personas y suponen el $38 \%$ de la población latinoamericana. Las mayores aglomeraciones son ciudad de México, con 20,2 millones; Sao Paulo, con 18,7 millones; Río de Janeiro, con 11,3; y Buenos Aires, con 11,7. Todas ellas se configuran como metrópolis de rango mundial.

En el año 2000, ciudad de México, con una población proyectada de 26 millones, y Sao Paulo, con 24 millones de habitantes, serán las dos aglomeraciones más grandes del mundo. Al mismo tiempo, según datos de las Naciones Unidas, Argentina, Chile y Uruguay tendrán una tasa combinada de urbanización del $88,6 \%$, por encima de la Europa del Norte, 88,3\%, Europa occidental, 81,2 \%, y Estados Unidos, 74,6\%.

De la segunda guerra mundial a nuestros días ha habido una verdadera explosión urbana. La tasa media anual de crecimiento de las ciudades de Latinoamérica ha ido del $4,4 \%$ en la década de los 60 , al $2,7 \%$ actual, y se prevé un índice del $2,2 \%$ para la primera década del siglo XXI, siempre muy por encima del resto del mundo, excepto en el caso del continente africano, con tasas de crecimiento medio anual de la población urbana que van del $4,3 \%$ en las primeras fechas considera-

' Informe Mundial sobre Asentamientos Humanos, 1986 (1989), Madrid, Ministerio de Obras Públicas y Urbanismo. 


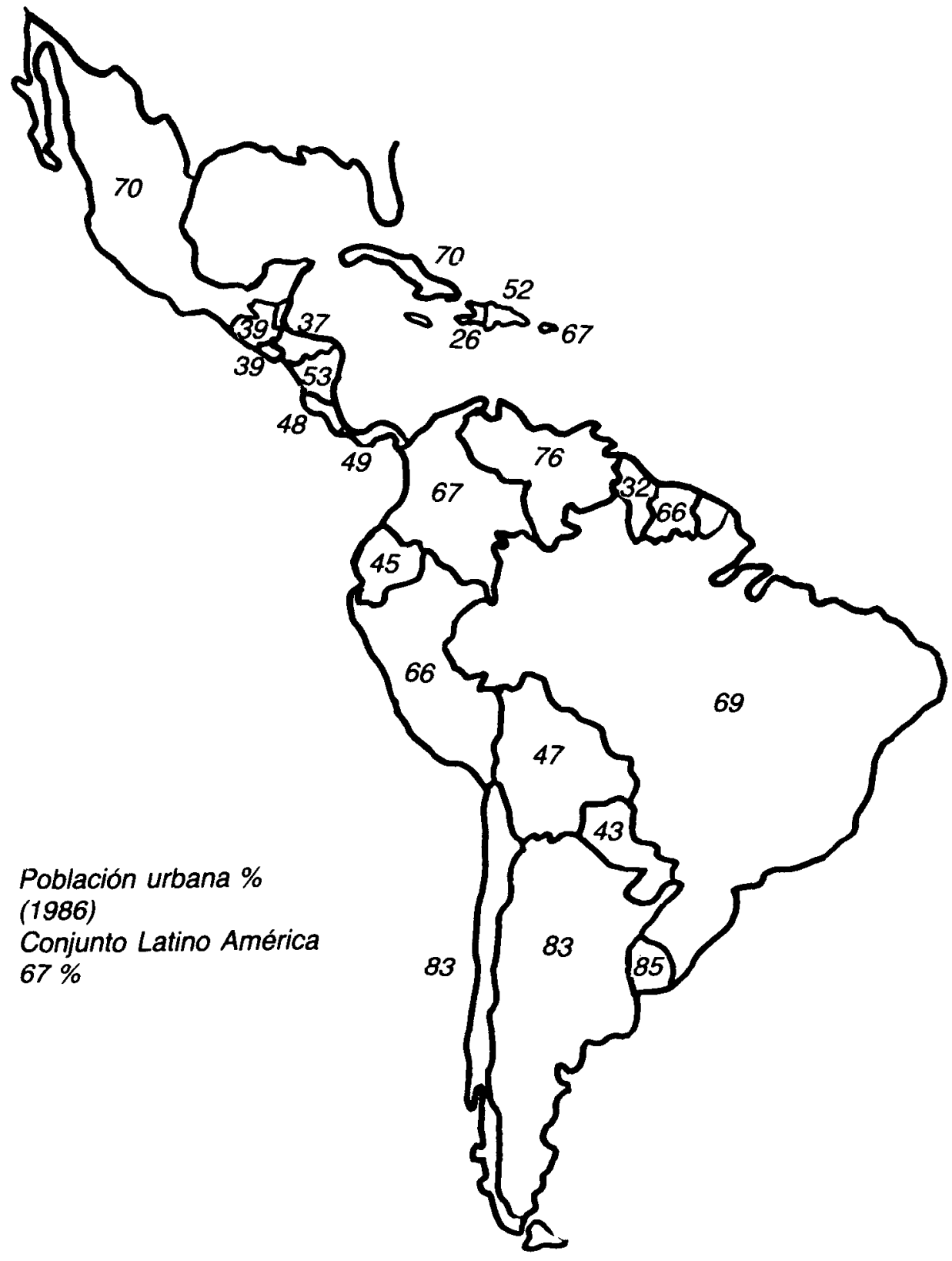

Figura 1.-Tasas de urbanización. 
das, al $4,9 \%$ actual, y a un $4,5 \%$ previsto para el periodo $2000-2010^{2}$. En esos mismos años, las tasas de crecimiento medio anual de la población urbana de los países desarrollados han sido respectivamente del 1,$0 ; 0,6 ;$ y 0,4 ; respectivamente.

Este espectacular crecimiento se explica por un fuerte crecimiento vegetativo $y$, sobre todo, por movimientos migratorios.

El crecimiento vegetativo es muy fuerte debido a unas tasas de natalidad elevadas, durante bastante tiempo en muchos países han oscilado entre el 30 y el 40 por mil, y a unas bajas tasas de mortalidad, en torno al 8 por mil, debido a los progresos de la medicina y mejora de la asistencia sanitaria. Excepto los países de la zona templada, con tasas de natalidad del 23,7 y de mortalidad del 8,3 por mil entre 1980 y 1985, más próximas a las de los países desarrollados, la mayoría de los latinoamericanos se encuentran aún inmersos en la etapa de crecimiento álgido que corresponde a la segunda fase del modelo de transición demográfica.

El fenómeno más importante ha sido el descenso de la mortalidad desde 1940. En unos 20 años se ha conseguido un control de la mortalidad que los países industrializados tardaron 70 u 80 años en conseguir. Las ciudades han sido las más beneficiadas por concentrar el personal y las inversiones sanitarias, de modo que la ciudad latinoamericana no ha sido hostil a la reproducción como lo fueron las ciudades europeas de finales del XIX y principios del siglo, la fertilidad es inferior a la rural pero alta.

Sin embargo, el mayor impacto en el crecimiento poblacional se debe al éxodo rural y movilidad entre ciudades de distinto rango. Así, en México, la migración es responsable de una cuarta parte del crecimiento de su población urbana, y en ciudad de México la migración proporciona más de la mitad de su crecimiento anual; mientras nacen 360.000 niños al año inmigran unas $\mathbf{4 0 0 . 0 0 0}$ personas.

Las ciudades se comportan como centros de cambio social y político que las nuevas clases dirigentes intentan impulsar y en principales focos de atracción que despierta esperanzas ocupacionales, de mejora económica y movilidad social para la inmensa mayoría de la población, sobre todo para los jóvenes de áreas rurales superpobladas, donde permane-

\footnotetext{
${ }^{2}$ Naciones Unidas: World Population Prospects as Assed in 1984. New York, 1986.
} 
cen las formas improductivas de tenencia de la tierra ${ }^{3}$. Sin embargo, con frecuencia el traslado de residencia no representa nada mejor que cambio de pobreza rural por urbana.

A diferencia de los países desarrollados, la industria no ha sido motor de urbanización, a menudo la industrialización es un proceso posterior e incompleto. No obstante, aunque no existe relación directa entre empleo industrial y urbanización, si hay asociación entre producción industrial y crecimiento urbano a través de denominado efecto demostración ${ }^{4}$ y del desarrollo de lo que Milton Santos denomina sector informal de la economía ${ }^{5}$. La industria y el comercio artesanal muestran extraordinaria capacidad en suscitar empleos no productivos, sustituyendo el aumento de la productividad técnica por el empleo de mano de obra barata y abundante. Simultáneamente las ciudades son centros administrativos cuyos organismos proporcionan multitud de empleos a través de puestos que no responden a necesidades objetivas sino al mero interés por crear una verdadera maraña de relaciones de dependencia basada en la influencia personal.

De este modo, Castells dice: "La urbanización en América Latina no es el reflejo de un proceso de "modernización", sino la expresión, a nivel de las relaciones socio-espaciales, de la agudización de las contradicciones sociales inherentes a su modo de desarrollo determinado por su dependencia específica dentro del sistema capitalista monopolista»" .

A todas estas causas de urbanización hay que añadir la que resulta de la tradición e importancia que ya tenía el fenómeno urbano antes de los años cincuenta y de la actual revolución tecnológica.

Las ciudades tuvieron un papel importante desde hace más de 1.500 años. Las principales culturas precolombinas, maya, azteca e inca, desarrollaron sistemas urbanos interiores con ciudades como puntos focales de interacción social, centros administrativos, religiosos y de comercio. Se apoyaban sobre hinterlands con densas poblaciones rurales que practicaban una agricultura intensiva de regadío. A menudo, las ciudades eran en su organización espacial una recreación cultural del cosmos.

${ }^{3}$ Brian, J. L., Berri: Consecuencias Humanas de la Urbanizacion. Madrid, 1975. Ed. Pirámide.

${ }^{4}$ Hirschman, A.: The Strategy of Economic Development. New Haven, 1958. Yale University Press.

${ }^{5}$ Milton Santos: O Espaco Dividido. Río de Janeiro, 1978 Livraria Francisco Alves Editora S A, p. 23-43.

${ }^{6}$ Castells, M.: La cuestión urbana. Madrid, 1979, 6. ${ }^{a}$ ed., siglo XXi, p. 78. 
Tenochtitlan, principal centro azteca, y Cuzco, capital inca, superaron en grandiosidad y tamaño a muchas de las ciudades del viejo mundo.

Luego, la colonización española y portuguesa introdujeron y desarrollaron un modelo urbano desplazado hacia la periferia que constituye la base del actual. El territorio se explota y organiza a partir de jerarquías urbanas que concentran el control administrativo y económico dentro de las primeras relaciones de dependencia en el marco del capitalismo periférico.

Modernizaciones sucesivas, como son las correspondientes al modelo económico exportador hacia afuera y de crecimiento hacia adentro, que se extienden desde los años ochenta del siglo pasado hasta los años cincuenta del nuestro, consolidan el fenómeno urbano dentro de nuevas formas de dependencia. Se crean algunos nuevos centros y se refuerzan los viejos polos históricos como Buenos Aires, Santiago de Chile o Lima.

\section{REDES URBANAS DESIGUALMENTE DISTRIBUIDAS EN EL ESPACIO}

A pesar de los elevados índices de urbanización los asentamientos se distribuyen de forma muy irregular por el territorio y en general forman redes muy desequilibradas espacialmente, incluso a nivel nacional. Tal como ponen de relieve los valores del índice $\mathrm{Rn}$, que mide la distribución espacial de los asentamientos a partir de sus distancias promedio con relación a los núcleos más próximos en un territorio de superficie conocida, y proporciona valores extremos de máxima concentración, 0 , a mayor dispersión, 2,15, las ciudades tienden a una disposición concentrada, con vacios interiores y fuerte desconexión entre ellas, lo que a la hora de la independencia propició la fragmentación política en múltiples Estados y ahora dificulta los flujos entre ellos y obstaculiza el desarrollo económico, social y cultural de los distintos países (Figura 2).

En este contexto, Lasuen, Friedman y otros han valorado especialmente la importancia de la distribución espacial de los núcleos urbanos en relación con el desarrollo económico ${ }^{7}$. Se considera que las innovaciones materiales, tecnológicas, mentales e institucionales que propician el desarrollo económico se canalizan a través de la jerarquía urbana,

${ }^{7}$ LASUEN, J. R.: "Urbanisation and Development: The temporal Interaction between Geographical and Sectorial Clusters". Urban Studies, vol. 10, n. ${ }^{\circ}$, junio, 1973, p. 163-188. 
Notas sobre el modelo urbano latinoamericano

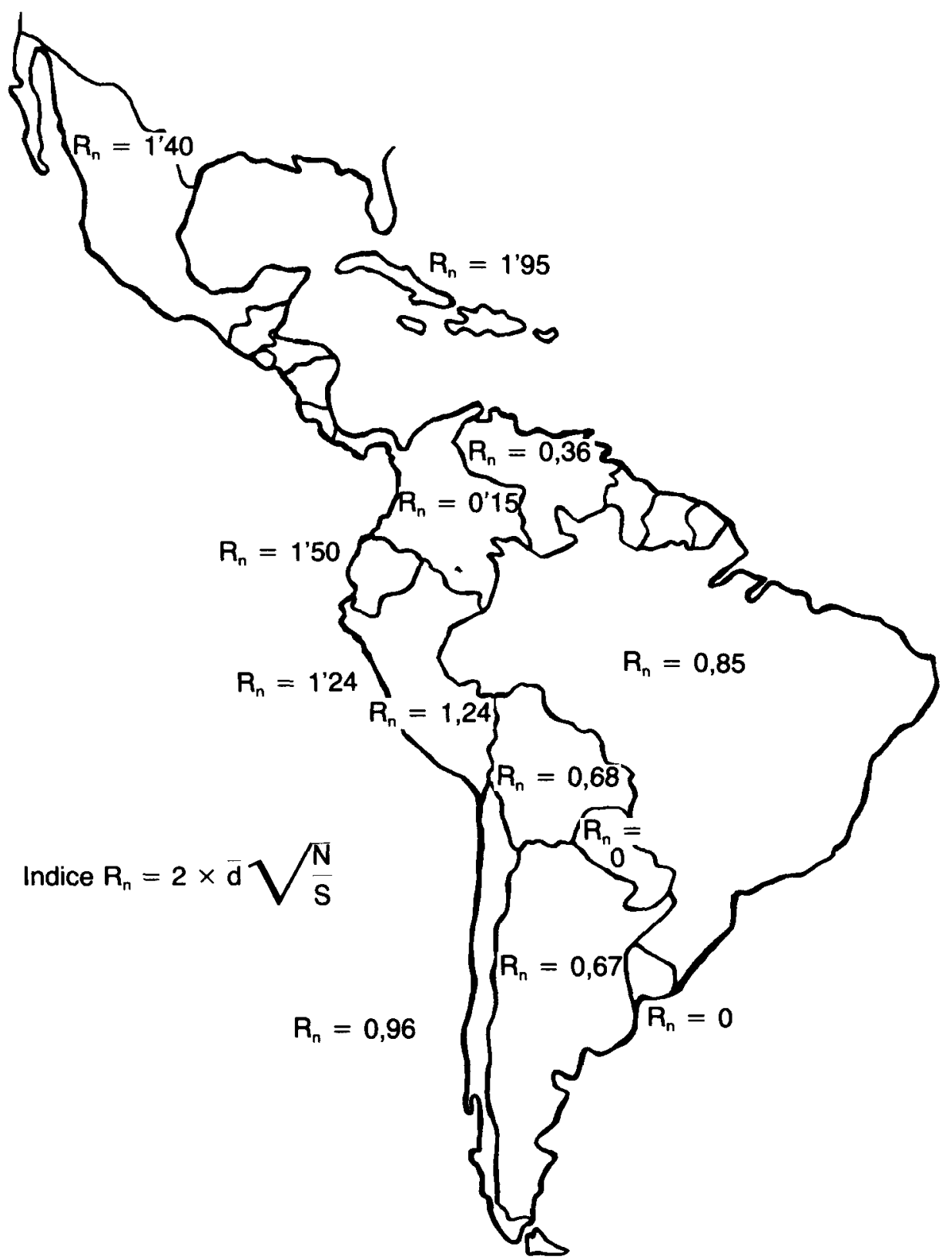

Figura 2.-Distribución espacial urbana.

273 
desde los centros de mayor rango hacia los de menor nivel. Por eso Racionero ${ }^{8}$ se preocupó por la forma de los sistemas urbanos, estableciendo una tipología que en lo referente a América Latina resulta del siguiente modo:

1. Concentrados: Es el caso de Argentina y Chile. Las ciudades se agrupan en una zona del territorio, no lo cubren en su totalidad por lo que son perjudiciales para el desarrollo económico.

2. Lineales: Es la disposición que adoptan las ciudades en Venezuela; se disponen a lo largo de un eje, aquí el norte del país, que concentra el desarrollo en contraste con el resto del país.

3. Dendríticos, como en Brasil: Las ciudades se sitúan franjas perpendiculares a la costa, siempre con una gran ciudad puerto que en el pasado facilitaba los intercambios con la metrópoli. Es perjudicial para el desarrollo, ya que el espacio geográfico y económico queda fragmentado en ejes inconexos.

4. Aglomerados, como Colombia: Los principales asentamientos urbanos se concentran en varios puntos del país, formando constelaciones de ciudades. Son adecuados para facilitar los procesos de difusión, siempre que se aseguren las comunicaciones entre las diversas agrupaciones urbanas.

5. Dispersos: Las ciudades se distribuyen de manera aletoria, como en México y Cuba. Es la forma más adecuada para el desarrollo a condición de garantizar las comunicaciones entre aglomeraciones y de forma especial las de la costa.

Todas estas distribuciones espaciales son, básicamente, consecuencia de sucesivas modernizaciones y dependencias, pero sobre todo de la colonial, que supuso la incorporación a la estructura metrópoli-satélite en el marco del capitalismo mercantil y de la revolución de los transportes marítimos.

En el mundo hispano, la conquista supuso un esfuerzo de cristianización, al menos a título de justificación, por lo que las primeras ciudades se ubicaron en emplazamientos precolombinos, en áreas de fuerte poblamiento indígena utilizado como mano de obra en las tareas productivas. Algunos de estos asentamientos, como Bogotá o ciudad de México, so-

${ }^{8}$ Racionero, L.: Sistema de ciudades y ordenación del territorio. Madrid, 1981, 2." ed. Alianza Universidad. 
bre anteriores ciudades, se han perpetuado a través del tiempo hasta hoy.

La existencia de recursos mineros motivó la creación de ciudades, a pesar de las dificultades impuestas por las condiciones climáticas, la altura o la ausencia de población indígena. Entre ellas figuran Potosi en Bolivia, Taxco o Zacatecas en México y Tegucigalpa en Honduras.

Por su parte la necesidad de dar salida a los productos mineros y agrícolas, y de asegurar las relaciones con la metrópoli primaron las localizaciones portuarias, como Santo Domingo en la República Dominicana, Acapulco y Veracruz en México, La Habana en Cuba, Guayaquil en Ecuador, Cartagena en Colombia, Balboa y Colón en el istmo de Panamá, Lima en Perú, y Salvador, Bahía y Río de Janeiro en Brasil. Especial importancia tuvieron Lima y Río de Janeiro al reunir la función portuaria y administrativa.

Por último, hubo ciudades especializadas en la función defensiva. Si al principio este carácter lo tuvieron todas, luego quedó restringido a ciudades en los límites del Imperio, en la linde con poblaciones belicosas como es el caso de Santiago de Chile con relación a los araucanos.

En el mundo portugués, la red urbana fue desarrollada sobre la base de la rentabilidad del intercambio de productos y la explotación intensiva de las zonas próximas a los puertos. Las ciudades contaron con el estímulo de las autoridades, la participación de los capitalistas privados y de los colonos. El carácter de esta colonización propició la mayor conexión entre los asentamientos y en consecuencia la formación de un solo Estado en el momento de la independencia.

Tras la independencia, la red urbana colonial, volcada en la periferia frente a los sistemas urbanos precolombinos, experimentó pocas modificaciones. Tan sólo las que resultan de la creación de nuevas ciudades en frentes pioneros, como Patagonia, el Mato Groso o la Guayana, o de la formación de redes regionales en Antioquía o La Pampa a partir de finales del siglo $X I X$ y principios del XX, coincidiendo con la mundialización de la economía, la consolidación de las bases del desarrollo desigual, las exportaciones de materias primas agrícolas y mineras al exterior, la afluencia masiva de inmigrantes y de capitales, y la primera industrialización. Los países más beneficiados de la nueva coyuntura, como Argentina y Uruguay, y en menor medida, Cuba y Chile, iniciaron un proceso de urbanización que elevó su población urbana a principios de siglo a tasas comparables a las de Europa (Figura 3).

El modelo económico actual, emanado de la revolución tecnológica y del consumo, que marginaliza la periferia al internacionalizar el con- 


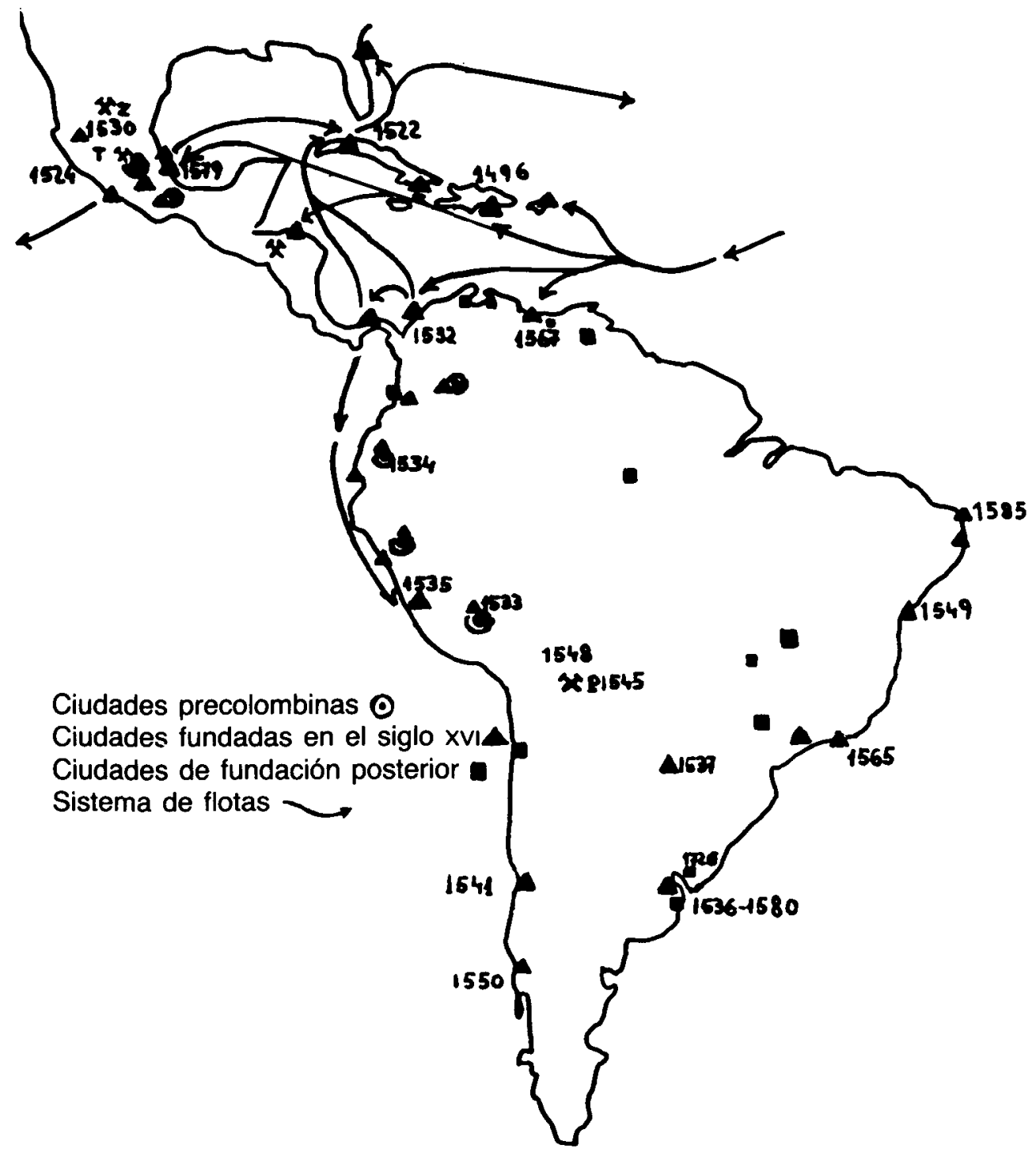

Figura 3.-Origenes de la Red urbana. 
sumo imitativo y no permitir la participación de los países periféricos en la innovación tecnológica y el intercambio de los centros, con su consiguiente endeudamiento y desencadenamiento de una feroz crisis económica y financiera desde 1982, consolida el modelo urbano anterior y agudiza sus contradicciones. En general se potencian los efectos de ciudad primada y de urbanización desigual. A orillas del Plata se forma en la actualidad una megalópolis que contrasta con los grandes vacíos de América del Sur, y ciudad de México y Sao Paulo se convierten en las mayores aglomeraciones del planeta.

\section{MACROCEFALIA Y REDES URBANAS INCOMPLETAS}

La aplicación de la regla rango-tamaño y del índice de primacía permite expresar de manera gráfica los grandes desequilibrios del sistema de ciudades en su distribución jerárquica.

Excepto Ecuador y Brasil, sistemas bicéfalos (Figuras 4, 5, 6, 7, 8 y 9 ) todos los demás son macrocéfalos. Una ciudad principal concentra la mayor parte de la población y de las actividades económicas del país por las ventajas locacionales derivadas de las economías externas, de escala y aglomeración, por la posición estratégica dentro de la red moderna de comunicaciones, por la tendencia a continuar la situación de dominio una vez establecida, por los beneficios que suponen la existencia de capitales e infraestructuras, y por las inversiones prioritarias del Estado.

Esta macrocefalia, fruto del modelo urbano heredado, ha sido reforzada en casi todos los países después de la Segunda Guerra Mundial, tal como se puede observar en los mapas (Figura 10 y 11), por el impacto de la revolución tecnológica, del consumo y la información, que han impulsado la industrialización dependiente e incompleta en Latinoamérica y el desarrollo de nuevas formas de producción y consumo. Los gobiernos, con su politica de crecimiento económico basada en la industrialización a cualquier precio, a la par que han facilitado la transnacionalización de la economía, el endeudamiento y la pérdida de capacidad, control y dirección de la economía por el Estado, han favorecido la macrocefalia al concentrar inversiones y facilidades en las aglomeraciones consolidadas. Por su parte, las industrias vinculadas al consumo doméstico buscan los emplazamientos más rentables en las ciudades principa- 

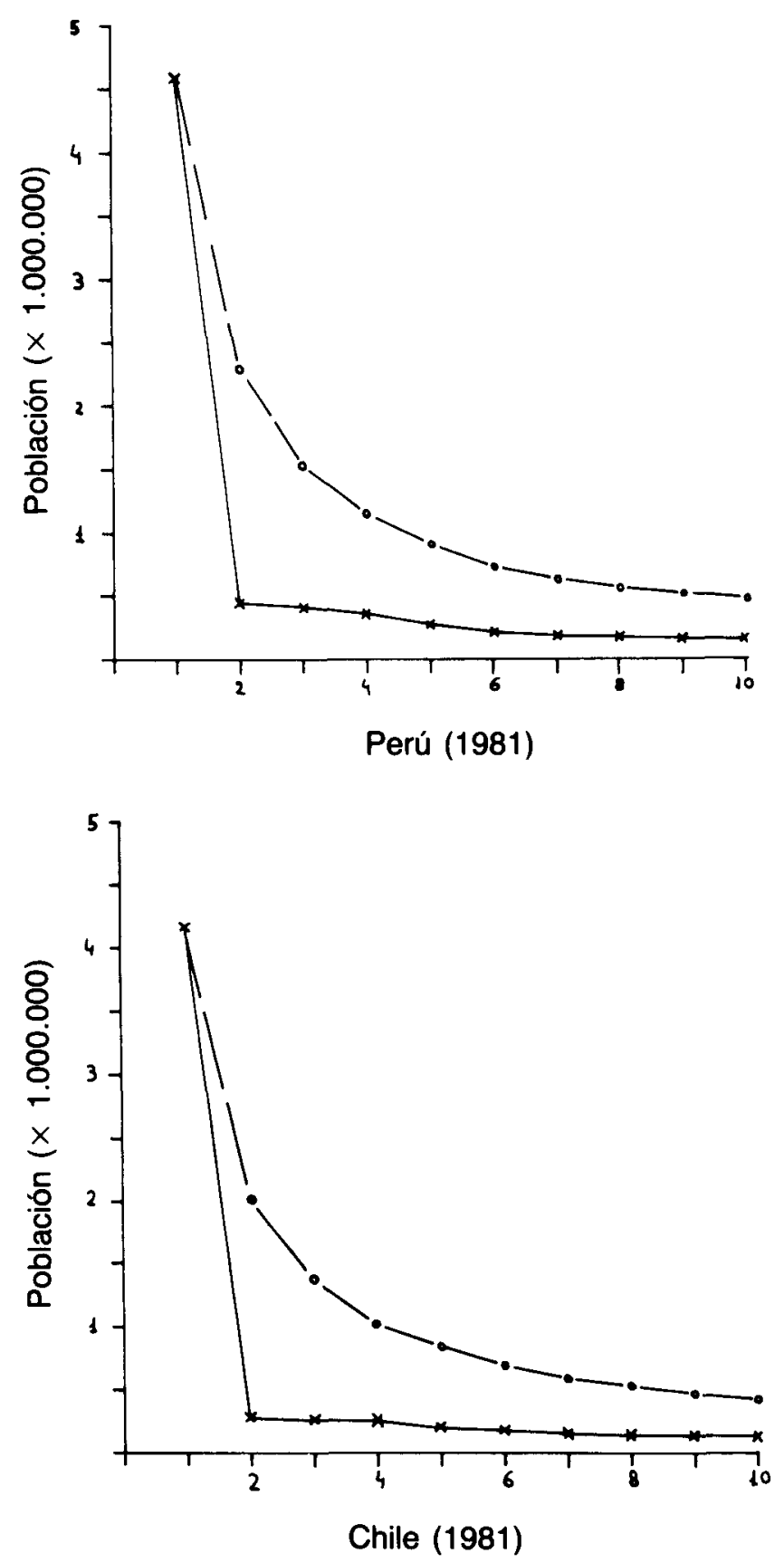

Figura 4.--Rango. Tamaño. 

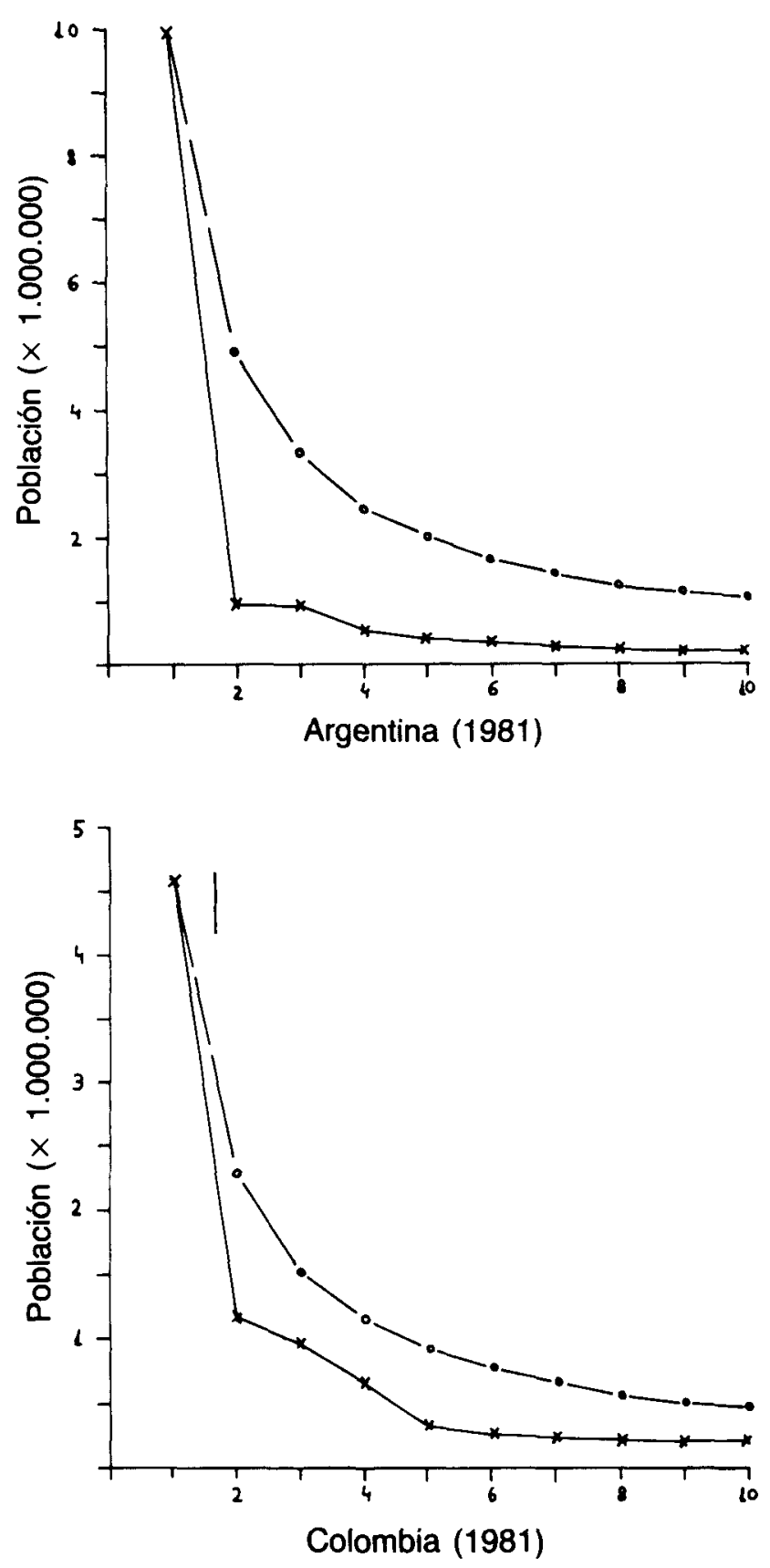

Figura 5.-Rango. Tamaño. 

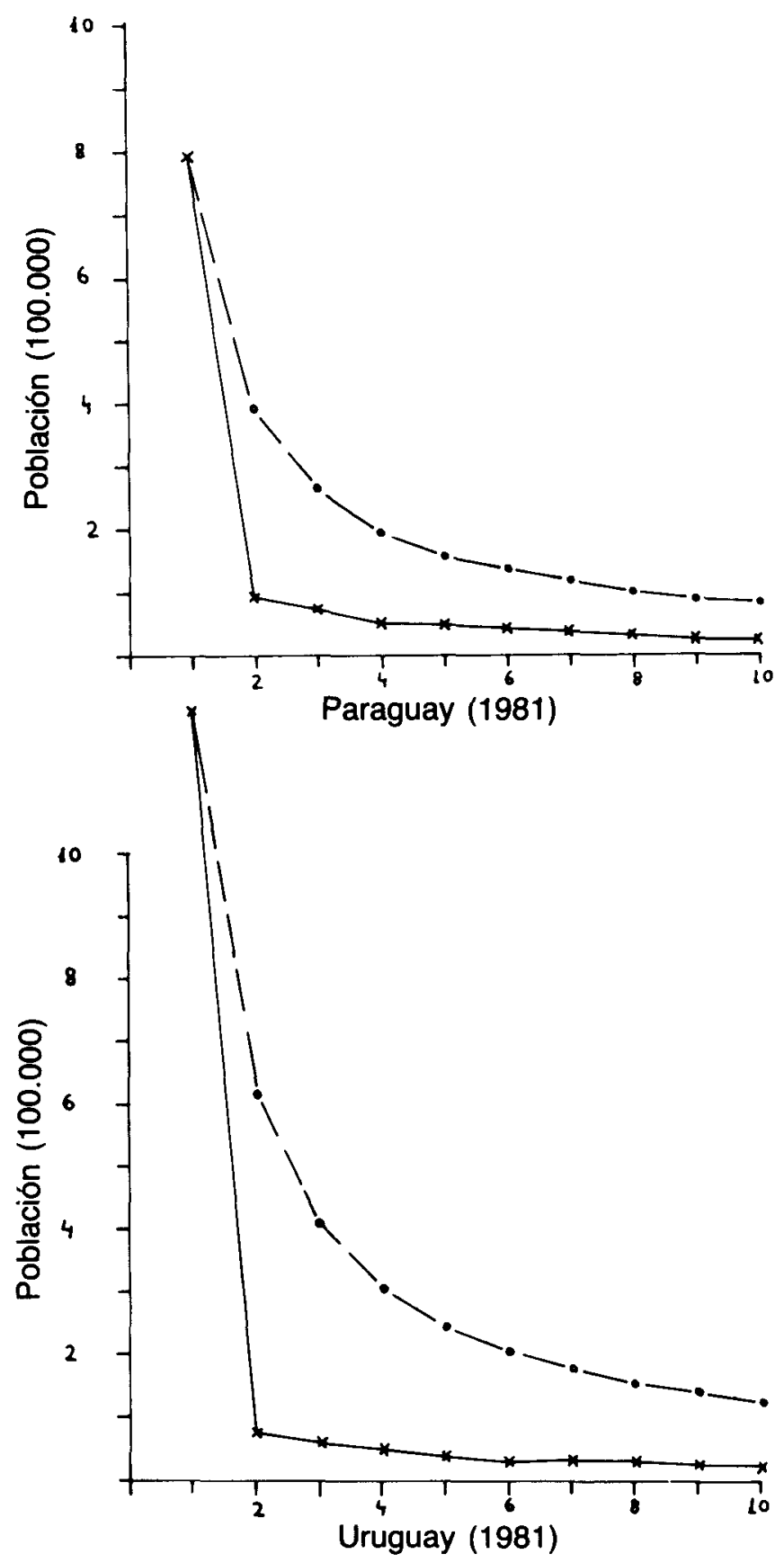

Figura 6.-Rango. Tamaño. 

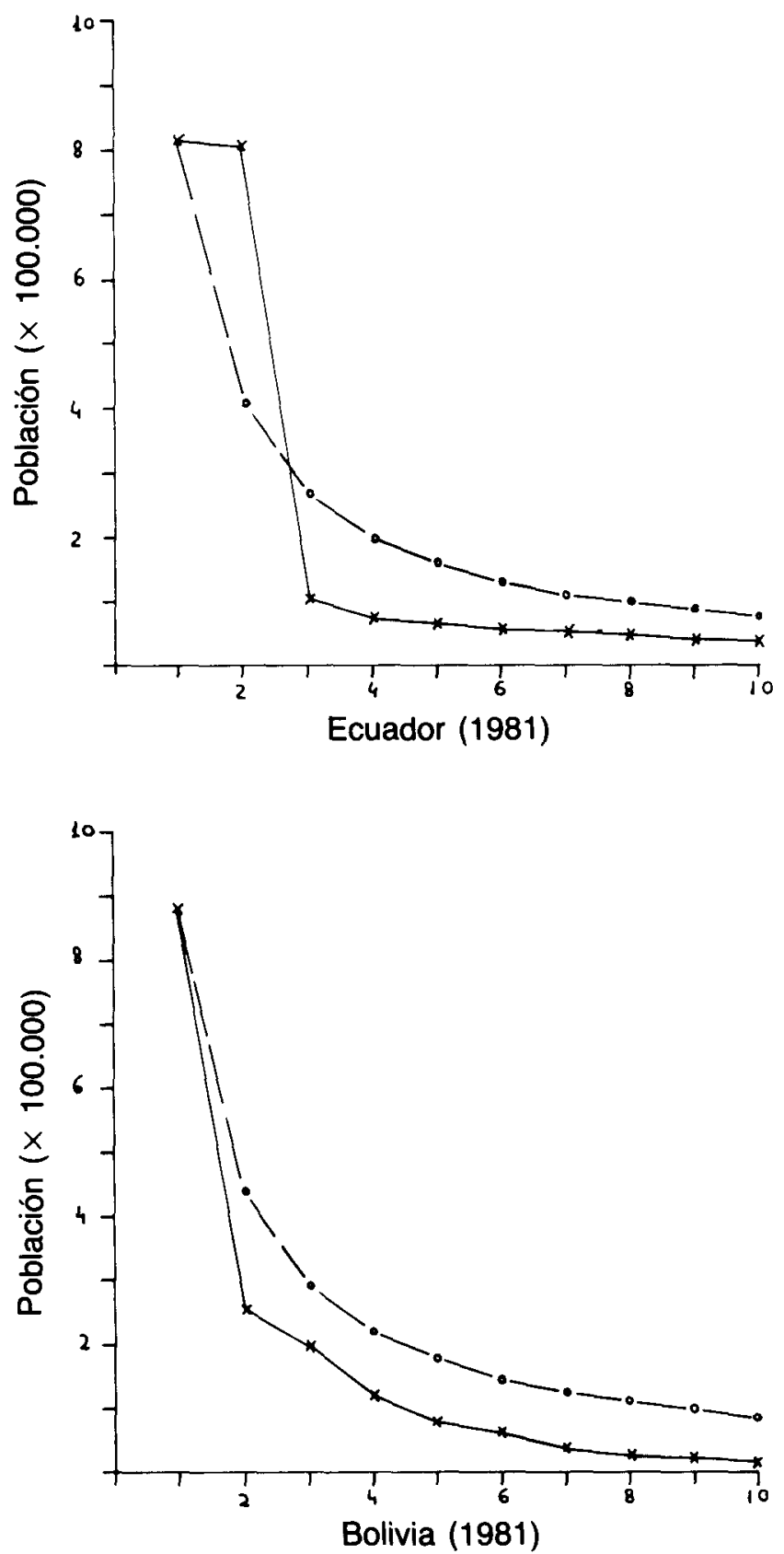

Figura 7.-Rango. Tamaño. 

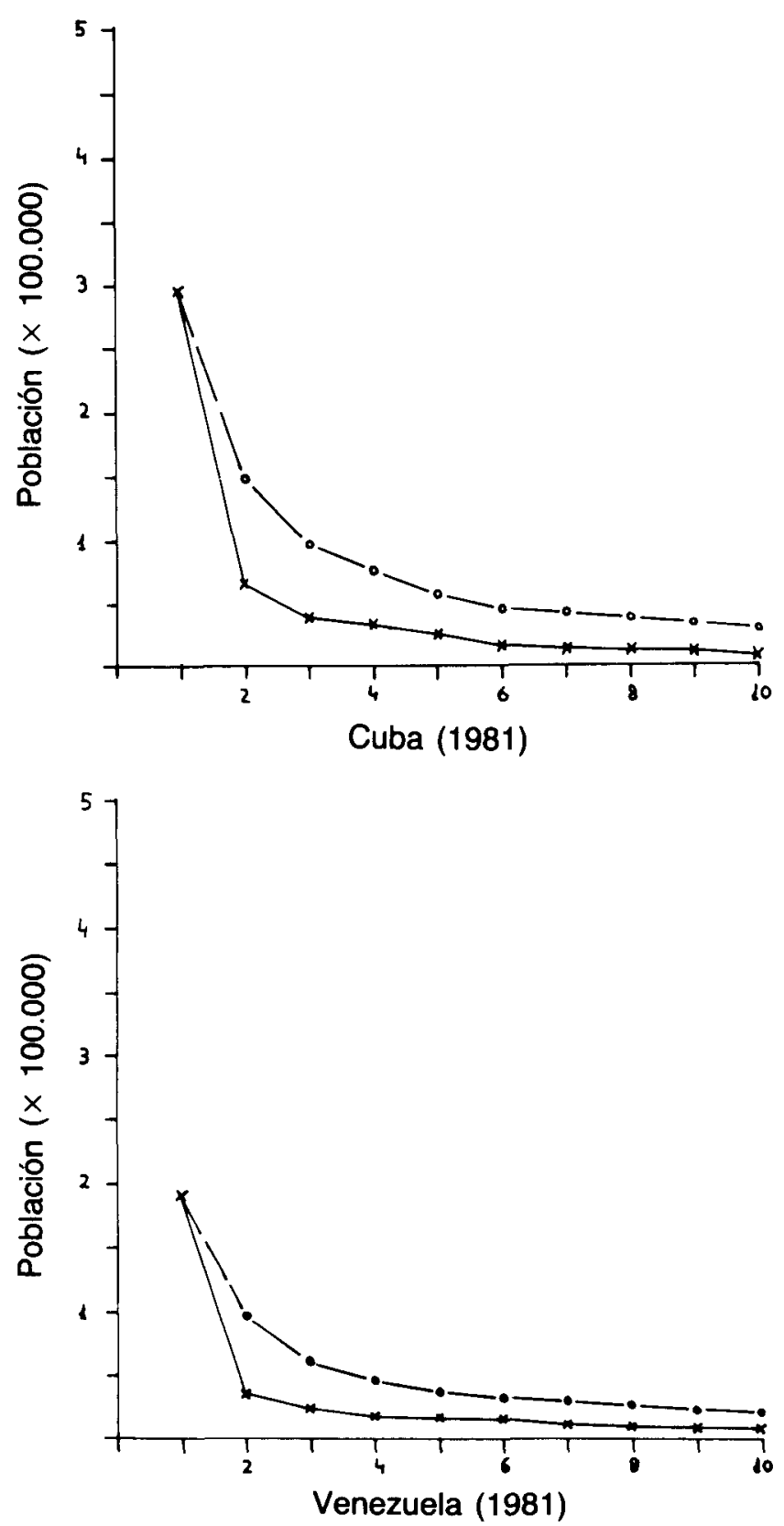

Figura 8.-Rango. Tamaño. 

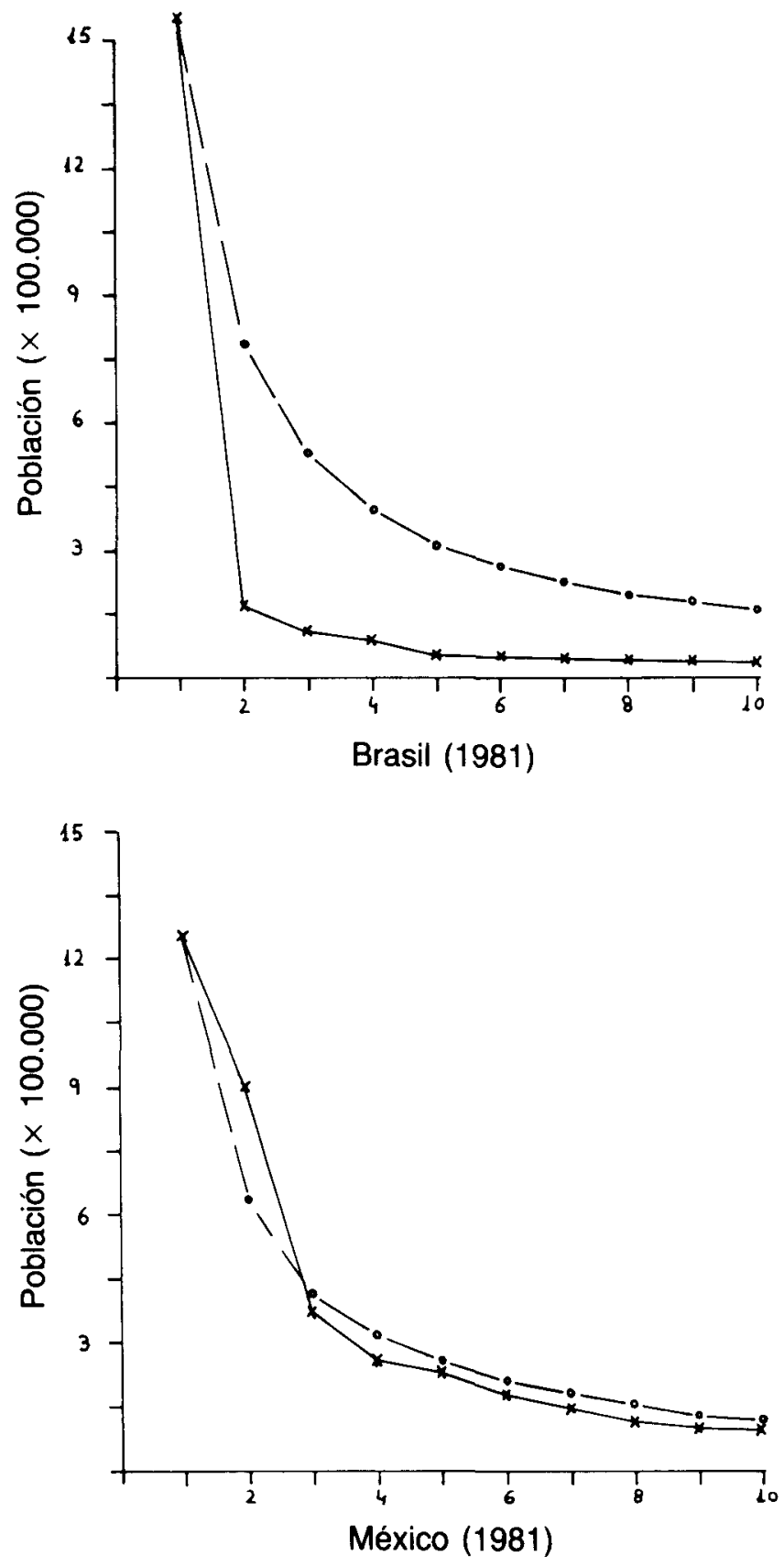

Figura 9.-Rango. Tamaño. 


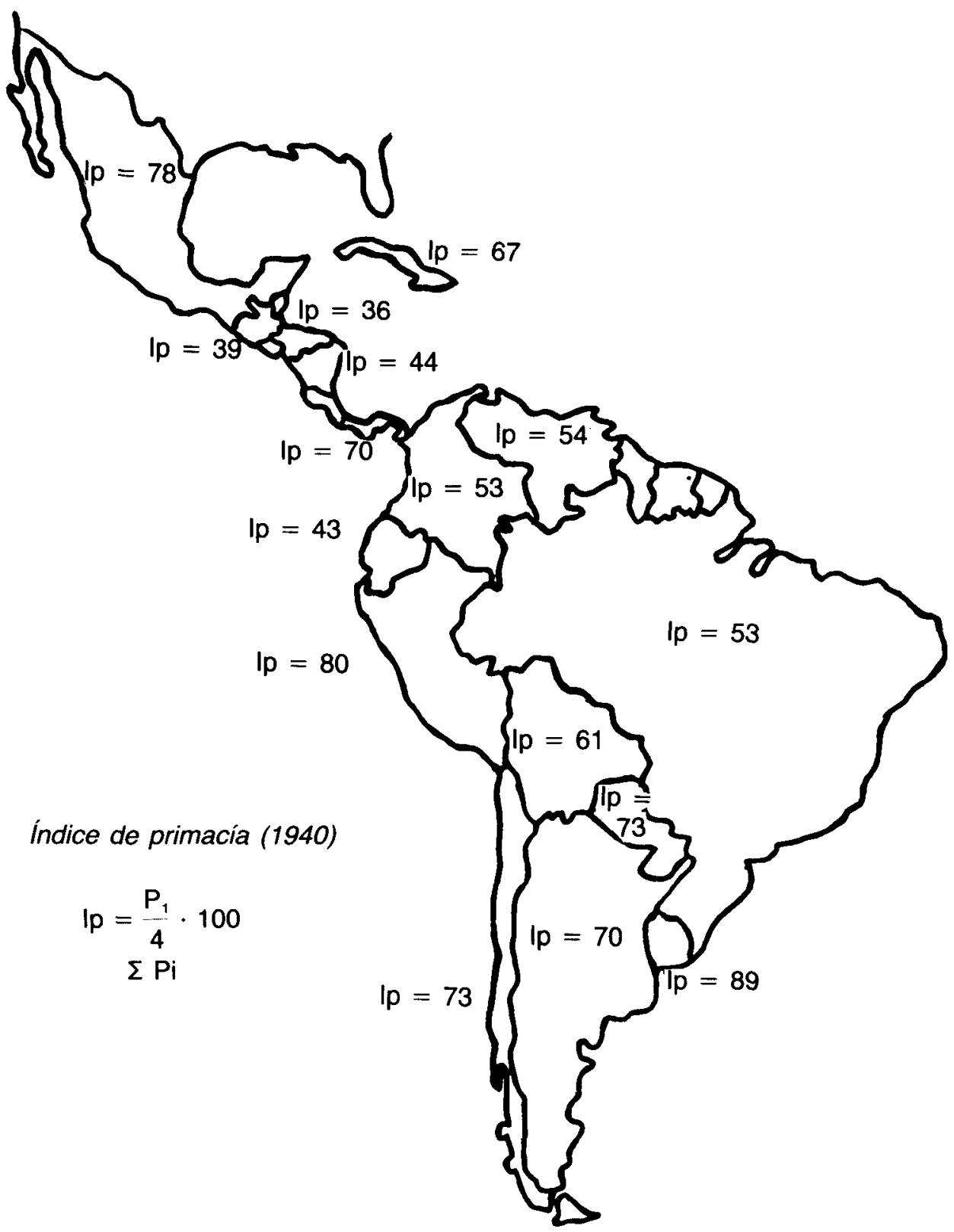

Figura 10.-Macrocefalia urbana. 


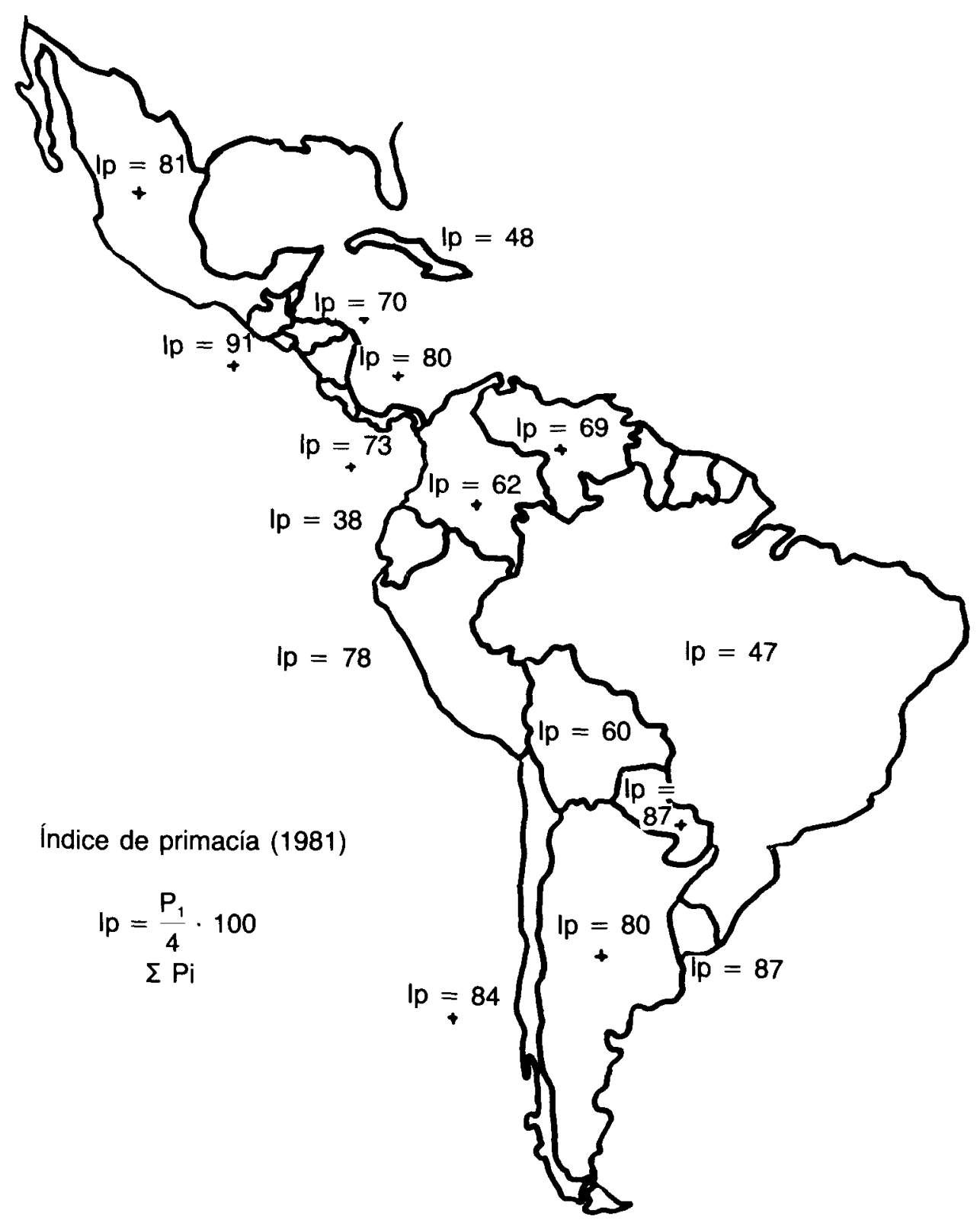

Figura 11.-Macrocefalia urbana. 
les y las mismas industrias de exportación acuden con frecuencia a esos mismos emplazamientos.

El resto de las ciudades se sitúan muy por debajo en población y actividad de lo que cabria esperar según la distribución regular y continua prevista por la regla rango tamaño. Sólo Brasil y Cuba muestran una distribución actual más próxima a la regla rango-tamaño y la primacía de sus ciudades principales ha disminuido desde los años cuarenta a nuestros días. En el caso de Brasil es consecuencia de una evolución hacia la formación de una red urbana más equilibrada y completa que tiende a acercarse al modelo de los países desarrollados a partir de una distribución histórica más regular de los asentamientos y de una decidida política correctora de los desequilibrios espaciales. En el caso de Cuba, se explica por una política de planificación autoritaria, inspirada en el desurbanismo soviético, que busca controlar el crecimiento y evitar la contradicción campo-ciudad a través de la limitación del tamaño de los asentamientos y la canalización de las inversiones industriales. En cambio, la macrocefalia se ha incrementado en casi todos los demás paises y de forma espectacular en América Central.

Resultan así redes urbanas jerárquicamente incompletas, escasamente integradas y con una tendencia piramidal imperfecta: a la cabeza se sitúan las aglomeraciones principales, metrópolis completas, que irradian su influencia sobre un vasto territorio, capaces de responder a las necesidades económicas y sociales de la población con medios propios, son los polos económicos principales; a continuación, ciudades regionales, algunas con carácter de metrópoli incompleta, con un área de influencia muy extenso pero que sólo pueden ejercer funciones comparables a las de las metrópolis completas a partir de contribuciones externas, en general procedentes de éstas; y ciudades locales, dispersas por el territorio y, a menudo, en declive por su aislamiento y mala conexión con el sistema de transportes.

El carácter de redes incompletas y desconectadas se pone también de relieve por la forma en que se efectúan las relaciones jerárquicas entre ciudades. A diferencia del mundo desarrollado es frecuente que las ciudades locales y regionales, de distinto rango poblacional y de equipamiento, no se relacionen con la de nivel inmediatamente superior para proveerse de bienes y servicios sino que acuden directamente a la principal. 


\section{UNA ESTRUCTURA URBANA EN MUTACIÓN}

El proceso reciente de urbanización está produciendo un fuerte impacto en la morfología y estructura de las ciudades latinoamericanas. Sus principales manifestaciones son:

1. La expansión de los centros de actividad, con una tendencia al aprovechamiento intensivo del suelo, que se manifiesta en un desarrollo en altura con transformación de la vieja trama y en la intensidad de fenómenos de substitución y cambio funcional en beneficio de un terciario y cuaternario altamente especializados.

2. Deterioro y abandono de los centros históricos, víctimas de la insuficiencia o ausencia de legislación protectora de ellos, y condicionados a menudo por emplazamientos en penínsulas, como La Habana, Montevideo y Panamá o semi islas, como Cartagena de Indias, que propician su aislamiento. Son frecuentes los casos de hacinamiento, ocupados por poblaciones de escasos recursos, como en Quito, La Paz, Lima, Bogotá..., pero tampoco faltan los ejemplos de gentrificación, como Olinda, municipio de la región metropolitana de Recife, o San Juan de Puerto Rico ${ }^{9}$. Algunos centros históricos, como los de las ciudades mexicanas próximas a la frontera, tienden a ser ocupados por clases altas extranjeras que buscan la calidad ambiental de estos espacios centrales.

3. Formación de espinas a modo de sectores de calidad desde el centro a los bordes de la ciudad ${ }^{10}$. Prolongan las actividades del CBD o centro de actividad, concentran servicios de calidad, las mejores zonas residenciales y el mejor equipamiento urbano, en contraste con el resto de la ciudad que se caracteriza por la insuficiencia de infraestructura ante la imposibilidad de las autoridades municipales por hacer frente a las necesidades de crecimiento de unas ciudades que caminan hacia lo que Ives Lacoste denomina "implosión urbana» ${ }^{11}$.

9 HaRDoY, J. E.: "Notas sobre las causas del abandono de los centros históricos de América Latina". Historia y futuro de la ciudad Iberoamericana. Madrid, 1985, CSIC, p. 89 122.

10 Griffin, E., y LaRRY, F.: «A model of Latin American City Structure". Geographical Review, 70, 1980, p. 397-422.

"LAcoste, Y.: "L'implosion urbaine". Hérodote. L'implosion urbaine?, n. 31, París, 1983 , p. 3-8. 
4. Proliferación de asentamientos marginales en terrenos ocupados ilegalmente: zonas de paso público, barrancos empinados, lechos secos o zonas de crecida de ríos. Las barriadas de Lima, los ranchos de Caracas, las villa miseria de Buenos Aires o las favelas de Río de Janeiro ocupan enormes extensiones. Más de la mitad de la población de Lima, Bogotá, Guayaquil y muchas otras aglomeraciones reside en asentamientos marginales y urbanizaciones piratas.

Las causas de la marginalidad residencial no se hallan tanto en situaciones ocupacionales como en la imposibilidad de la mayoría de la población para acceder a viviendas privadas y en la incapacidad de la economía de mercado y del Estado para proporcionar servicios públicos adecuados a ciudades de más de 100.000 habitantes que han crecido a un ritmo del $6 \%$ anual. Sao Paulo y México han tenido períodos de crecimiento anual del $8 \%$ al $12 \%$, lo que supone el doblamiento de la población en seis ó nueve años. Por eso los organismos internacionales aconsejan hoy la ayuda a la autoconstrucción de la vivienda y la regulación de los asentamientos espontáneos en lugar de su destrucción como fue práctica normal en el pasado.

A menudo las barriadas han sido foco crucial de movilizaciones populares, como lo fueron en Santiago de Chile entre 1965 y 1973, o en ciudad de México y Monterrey, entre 1970 y $1976^{12}$, pero en general constituyen más un vehículo de integración social y económica de los sectores populares urbanos que de cambio político.

5. Problemas de congestión de tráfico, polución, suministro de agua e insuficiencia de alcantarillado, que adquieren especial gravedad en las principales aglomeraciones por la concentración de la población y de la industria. Ciudad de México es, hoy, la ciudad más contaminada del mundo, dos millones de habitantes carecen de agua corriente en sus viviendas y el $20 \%$ de las casas no tiene alcantarillado. Por eso la planificación ecológica y la protección ambiental se convierte en políticas prioritarias de actuación para los poderes públicos, pero a la insuficiencia de los presupuestos por el continuo crecimiento de las ciudades hoy se añaden las dificultades derivadas de la deuda pública.

12 Castells, M.: La ciudad y las masas. Sociologia de los movimientos sociales urbanos. Madrid, 1986, Alianza Universidad. 


\section{LA URGENCIA DE LA PLANIFICACIÓN}

Los problemas derivados del crecimiento urbano incontrolado y de la debilidad de las redes urbanas fuerzan a los poderes públicos a planificar e intentar el control del crecimiento a nivel local y regional, a través de diferentes políticas.

En todas partes se aprueban Leyes Nacionales de Reforma Urbana y Planes Nacionales de Desarrollo Urbano; surgen instituciones de desarrollo municipal, como la Fundación Para el Desarrollo de la Comunidad y Fomento Municipal, creada en Venezuela para dar asistencia técnica y financiera a los gobiernos locales y asentamientos urbanos de bajas rentas.

A nivel regional, las principales actuaciones se han dirigido a la creación de ciudades nuevas y al establecimiento de centros y polos de desarrollo en ciudades de tamaño medio ya existentes que debían actuar como centros de atracción y contrarrestar la acción de las grandes ciudades. Para ello el Estado crea infraestructuras, fomenta las inversiones y concede incentivos fiscales a empresas y particulares. Así, en Brasil, con una constante preocupación por la integración política, social y económica, se procedió al traslado de la capital a Brasilia, se han construido un centenar de ciudades nuevas en el Sur, se han promovido polos de crecimiento en el Nordeste para impedir su despoblación y se construye la carretera transamazónica para estimular la creación de centros de crecimiento a lo largo de ella. En Colombia, se han establecido cuatro regiones económicas entorno a cada una de las cuatro principales ciudades como polo de crecimiento: Barranquilla, en la costa Atlántica, Cali en el Suroeste, Medellín en el Noroeste y Bogotá en el Centro.

La creación de ciudades nuevas forma parte del proceso de planificación de centros de crecimiento. Entre las nuevas ciudades figuran: capitales, como La Plata, Belo Hozironte, Goiania, Brasilia; ciudades industriales, como Nuva Guayana o El Tablazo; ciudades gemelas o de descongestión, como Tuy, a $30 \mathrm{~km}$ de Caracas, o los proyectos de ciudades paralelas de Río de Janeiro, en la Bahía de Jacarepagua, a 20 $\mathrm{km}$, y de ciudad de México, entre Yutepec y Cuernavaca, en el Estado de Morelos.

A nivel local, los temas prioritarios son los de empleo, vivienda, suministro de agua, alcantarillado y polución. Los planes se conciben con grandes expectativas pero pocas veces se llevan a cabo. Los enfoques suelen ser excesivamente sectoriales y no se tiene en consideración la 
interacción entre el desarrollo de la ciudad y su interland ni los vínculos entre las ciudades.

La eficacia de la planificación, desbordada por la magnitud de los problemas y la insuficiencia de recursos económicos, es muy reducida en general. Los programas de Brasil o Colombia, dirigidos a crear nuevos polos de crecimiento y frenar el crecimiento de las metrópolis, han tenido un éxito limitado. El Plan Nacional de Desarrollo Urbano de México que tenía como objetivo fundamental la descentralización por concentración del crecimiento en nuevos asentamientos, entre ellos cuatro puertos industriales que habían sido designados como polos de crecimiento en 1973: Coatzacoalcos, Ciudad Madero, Salina Cruz y Lázaro Cárdenas, no ha podido evitar que la población y la actividad industrial siguiera concentrándose en las tres áreas metropolitanas de Ciudad de México, Guadalajara y Monterrey.

A nivel local, sin embargo, se pueden observar algunos logros parciales, como la mejora de la efectividad de la asistencia al desarrollo de los asentamientos urbanos a través del Banco Mundial, la asimilación del crecimiento poblacional desde los años setenta en Cali, Colombia, o la mejora de las favelas de Río mediante trabajos planeados, financiados y llevados a cabo por sus mismos vecinos.

El lento pero progresivo descenso del crecimiento anual de población urbana, con una tasa prevista del $1,7 \%$ para el año 2025 ; el mayor crecimiento de las ciudades medias con relación al de las metrópolis en los últimos años en casi todos los países; la consolidación de las ciudades nuevas, son sintomas que abren alguna esperanza a la corrección de los desequilibrios del modelo urbano latinoamericano, aunque para ello será preciso superar las contradicciones del capitalismo periférico, responsable de la crisis estructural de Latinoamérica y de su modelo urbano que es una de sus más claras manifestaciones sobre el territorio. 